\title{
Comparison of Conventional Methods for Diagnosis of Visceral Leishmaniasis in Children of the Center-West Region of Brazil
}

\author{
Yvone M. Brustoloni' ${ }^{1}$, Rivaldo V. Cunha ${ }^{2}$, Maria Elizabeth Dorval ${ }^{3}$, Elisa T. Oshiro ${ }^{3}$, Elenir R.J.C. Pontes ${ }^{4}$, Ana Lúcia L.Oliveira ${ }^{1}$, \\ Lilian Hillebrand ${ }^{1}$ and Luis Felipe Ribeiro ${ }^{1}$ \\ ${ }^{1}$ Department of Pediatrics, ${ }^{2}$ Department of Medicine, ${ }^{3}$ Department of Patology, ${ }^{4}$ Department of Public Health, \\ Federal University of Mato Grosso do Sul; Campo Grande, MS, Brazil
}

\begin{abstract}
In Brazil, sophisticated techniques currently employed for diagnosis of visceral leishmaniasis, such as polymerase chain reaction-based assays, are only available in major research centers, whereas conventional methods are still used in many areas where the disease occurs. In the state of Mato Grosso do Sul, in the country's Center-West Region, visceral leishmaniasis has recently emerged in many cities, and duration of the disease, from the onset of symptoms to diagnosis, has been short. Considering that results of diagnostic tests may depend on the phase of the disease, we compared direct examination of bone marrow aspirates (BMAs), BMA culture, and serology by Indirect Immunofluorescence Antibody Test (IFAT) for diagnosis in children, according to time of evolution ( $\leq 30$ days or $>30$ days) and to spleen size $(\leq 5 \mathrm{~cm}$ or $>5 \mathrm{~cm})$ at admission. Duration of the illness did not interfere with test positivity: direct smear examination and IFAT were positive in more than $80 \%$ of patients, as was culture in around $60 \%$. Results of positive microscopy, however, where predominant in patients with larger spleens. Thanks to the association of traditional techniques, only a few patients had to begin a treatment trial without confirming the diagnosis. Conventional methods for diagnosis of visceral leishmaniasis are still indispensable in our region, and training professionals in basic techniques should be incremented. The highest sensitivity in laboratory diagnosis among the cases investigated was that obtained with a combination of BMA direct examination and IFAT, nearing $100 \%$. Key-Words: Visceral leishmaniasis, kala-azar, diagnosis, direct microscopy, immunological tests.
\end{abstract}

Visceral leishmaniasis, or kala-azar, is a serious health problem in Brazil [1] and in many countries around the world [2]. Accurate diagnosis is an important tool in the control of the disease, regardless of geographical location. In recent years, sensitive and specific tests, as those based on molecular methods such as polymerase chain reaction (PCR) have been used to detect DNA of Leishmania in a variety of clinical samples [3-8]. Although these techniques constitute a promising advance for the diagnosis of visceral leishmaniasis, they are usually sophisticated and are not available in many places where laboratory facilities are limited.

In the state of Mato Grosso do Sul, situated in the Brazilian Center-West Region, diagnosis is still based on the use of conventional methods, such as the demonstration of parasites in stained slides or cultures of bone marrow aspirate (BMA) and the detection of antibodies in sera by the Indirect Immunofluorescence Antibody Test (IFAT) in patients with a suggestive clinical picture of visceral leishmaniasis. In this study, we analyzed the results of these techniques and compared them with the duration of the disease and with spleen size in children admitted to a reference hospital for treatment of visceral leishmaniasis. In addition, we attempted to identify the best combination of tests for diagnosis in this region of Brazil.

Received on 22 July 2006; revised 28 November 2006.

Address for correspondence: Dr. Yvone Maia Brustoloni. Av $1^{\circ}$ de maio, 528, Jardim São Bento. Zip code: 79004-620. Campo Grande, Mato Grosso do Sul, Brazil. Phone: (67) 3383-4063. Fax: (67) 3321-5852. E-mail : brustoloni@uol.com.br.

The Brazilian Journal of Infectious Diseases 2007;11(1):106-109. (C) 2007 by The Brazilian Journal of Infectious Diseases and Contexto Publishing. All rights reserved.

\section{Materials and Methods}

From January 1998 to February 2005, 116 children living in Mato Grosso do Sul were admitted to a reference hospital (NHU-UFMS) in Campo Grande, the state's capital city, presenting with manifestations compatible with visceral leishmaniasis (fever, hepatosplenomegaly, pancytopenia, hypergammaglobulinemia). BMAs were collected from all children by sternal puncture or, more rarely, from the iliac crest; BMA cultures were performed for 86 cases and IFAT for 79. When possible, BMA inoculation in hamster was obtained. Duration of the illness ( $\leq 30$ and $>30$ days) and spleen size on admission $(\leq 5 \mathrm{~cm}$ and $>5 \mathrm{~cm}$, measured from the lowest left rib to the distal edge of the organ) were recorded and crossed with the tests results. Sixty five patients were submitted to all three diagnostic procedures, and the combination of the methods in these children was analyzed.

\section{Direct Examination (BMA Microscopy)}

Amastigotes were detected by microscopic examination of Giemsa-stained slides. Four slides from each patient were analyzed with a $10^{\prime}$ eyepiece and a 100' oil objective. The entire smear was examined (more than 1000 microscopic fields) before a negative result was assigned.

\section{Culture}

BMAs were cultured in Novy-MacNeal-Nicolle (NNN) with Schneider's insect medium supplemented with $20 \%$ fetal calf serum, incubated at $24^{\circ} \mathrm{C}$ and weekly examined by microscopy for the presence of the parasite until, eight weeks.

Indirect Immunofluorescence Antibody Test (IFAT)

Performed with a commercial kit (Fiocruz-Bio-Manguinhos, 
Rio de Janeiro), using promastigotes as antigen. Titers $=1: 40$ were considered positive.

Statistics

Fisher's exact and $\chi^{2}$ tests were used to compare proportions. Statistical significance was defined as $\mathrm{p} \leq 0.05$.

Ethical Issues

Approval to conduct the study was obtained from the Ethics Committee of the Federal University of Mato Grosso do Sul.

\section{Results}

Of the 116 children with suspected visceral leishmaniasis on admission, $83.6 \%(n=97)$ had parasites detected by BMA direct examination. IFAT showed a positivity of $86.1 \%$ (68/ 79). BMA culture was the least sensitive method, confirming diagnosis in just $60.5 \%$ (52/86) of the samples tested.

In most patients referred from other hospitals for diagnosis confirmation and treatment, only direct microscopy had already been tried, and serology had been requested in a few cases.

As many as $70 \%$ of the children exhibited suggestive signs and symptoms for 30 days or less, and in 30\% the disease was diagnosed after 30 days. Table 1 shows the positivity of each method, according to the duration of the disease. No statistically significant difference was found when cases with shorter ( $\leq 30$-day) or longer ( $>30$-day) duration of the disease were compared for the three tests (direct microscopy, $\mathrm{p}=0.344$; culture, $\mathrm{p}=0.360$; IFAT, $\mathrm{p}=1.000$ ).

Spleen size larger than $5 \mathrm{~cm}$ on admission was found in $86.2 \%$ of the patients (100/116); $13.8 \%(16 / 116)$ had $5 \mathrm{~cm}$ or less. BMA direct microscopy showed a greater number of positive results when spleen size was larger than $5 \mathrm{~cm}(\mathrm{p}=$
0,024) (Table 2). No statistically significant difference was found between spleen sizes regarding the results of culture ( $\mathrm{p}$ $=1.000)$ or $\operatorname{IFAT}(\mathrm{p}=1.000)$.

It was not possible to submit all the 116 children to the three diagnostic methods. This was possible in 65 of them. Had direct microscopy or serology been used separately, as many as $15.4 \%$ of the patients would not have been diagnosed. If direct microscopy and culture were employed in association (without IFAT), $13.8 \%$ cases would remain without etiologic confirmation. If direct microscopy and serology (or all three methods) had been combined, only one patient (1.5\%) would remain without diagnostic confirmation at the time of admission; this child had parasites detected by hamster inoculation five months later. This emphasizes the importance of associating serology and BMA direct microscopy in order to reach higher positivity rates in diagnosis.

\section{Discussion}

An epidemic wave of visceral leishmaniasis has been triggered in the state of Mato Grosso do Sul, and children have been exhibiting an illness of short duration, from onset of symptoms to diagnosis, so that most cases are seen at the hospital when clinical and laboratory features are already striking. We thus chose to compare the positivity of conventional methods in the diagnosis of cases exhibiting these characteristics.

Demonstration of amastigotes in bone marrow smears ranges from $60 \%$ to $85 \%$ [9]. Brazilian researchers have reported rates from $69.3 \%$ to $81.2 \%$ in pediatric patients [1014]. However, some factors interfere with the positivity rates of the method. Bone marrow aspiration should be carried out by trained individuals, and performance of a correct technical procedure is vital to its sensitivity [15], since inadequate aspiration may provide insufficient or inadequate material

Table 1. Positivities of three conventional methods for diagnosis of visceral leishmaniasis in children according to the duration of the disease, NHU-UFMS, 1998-2005

\begin{tabular}{lccc}
\hline $\begin{array}{l}\text { Duration of the } \\
\text { disease (days) }\end{array}$ & $\begin{array}{c}\text { BMA microscopy } \\
\mathbf{N}^{\mathbf{0}} \text {.positive/n tested (\%) }\end{array}$ & $\begin{array}{c}\text { BMA Culture** }^{* *} \\
\text { N positive/n tested (\%) }\end{array}$ & $\begin{array}{c}\text { IFAT*** }^{* *} \\
\text { N positive/n tested (\%) }\end{array}$ \\
\hline$\leq 30$ & $66 / 81(81.5)$ & $35 / 61(57.4)$ & $47 / 55(85.5)$ \\
$>30$ & $31 / 35(88.6)$ & $17 / 25(68.0)$ & $21 / 24(87.5)$ \\
Total & $97 / 116(83.6)$ & $52 / 86(60.5)$ & $68 / 79(86.1)$ \\
\hline
\end{tabular}

* $\mathrm{p}=0.344\left(\chi^{2}\right.$ test $){ }^{* *} \mathrm{p}=0.360\left(\chi^{2}\right.$ test $) * * * \mathrm{p}=1.000$ (Fisher's exact test).

Table 2. Positivities of three conventional methods for diagnosis of visceral leishmaniasis in children according to the spleen size on admission, NHU-UFMS, 1998-2005

\begin{tabular}{lccc}
\hline Spleen size (cm) & $\begin{array}{c}\text { BMA microscopy * } \\
\text { N positive/n tested (\%) }\end{array}$ & $\begin{array}{c}\text { BMA Culture** } \\
\text { N positive/n tested (\%) }\end{array}$ & $\begin{array}{c}\text { IFAT*** } \\
\text { N positive/n tested (\%) }\end{array}$ \\
\hline$\leq 5$ & $10 / 16(62.5)$ & $6 / 10(60.0)$ & $7 / 8(87.5)$ \\
$>5$ & $87 / 100(87.0)$ & $46 / 76(60.5)$ & $61 / 71(85.9)$ \\
Total & $97 / 116(83.6)$ & $52 / 86(60.5)$ & $68 / 79(86.1)$ \\
\hline
\end{tabular}

* $\mathrm{p}=0.024$ (Fisher's exact test) ${ }^{* *} \mathrm{p}=1.000$ (Fisher's exact test) ${ }^{* * *} \mathrm{p}=1.000$ (Fisher's exact test). 
(peripheral blood). Expertise of the microscopist, number of fields analyzed, and time spent to examine smears are other significant aspects [16]. Sensitivity is decreased when BMAs are quickly examined, but, when they are examined for appropriate lengths of time, examination can achieve a sensitivity level close to that of spleen aspirates (> 95\%) [16].

Finally, parasite tissue burden is another important aspect. In our study, although parasites were scarce in the early phases of the disease, the expertise and persistence of the microscopist allowed positive results to be achieved; some smears in fact required inspection of a large number of fields, and parasite numbers, in some cases, were as low as one or two in the entire slide. Therefore, parasite tissue burden is another factor influencing the positivity of direct microscopy; in visceral leishmaniasis, a cytokine mediated cell-type response defines patients as scarcely, moderately, or heavily parasitized [17].

Spleen size larger than $5 \mathrm{~cm}$ on admission was associated with a greater number of positive results by direct microscopy. On the other hand, when spleen size is small $(\leq 5 \mathrm{~cm})$, IFAT may be a better alternative for diagnosis.

Although culture was the least sensitive method in the study (60.5\%), the sensitivity of culture may vary from $40 \%$ to $50 \%$ when BMAs are used, and from $70 \%$ to $98 \%$ with the use of splenic aspirates [18]. The main difficulty with cultures is the high contamination rate in the early stages $[18,19]$. Moreover, culture-based diagnosis may take days or weeks and so, is not appropriate for the purpose of deciding to begin a treatment.

A wide range of serological methods is available for diagnosis of visceral leishmaniasis $[18,19]$. Among the several serological techniques employed, Indirect Immunofluorescence is one of the most widely used in Brazil [1]. ELISA is a more sensitive test [1] and may use highly sensitive recombinant antigens such as rK39 [19]; the direct agglutination test (DAT) is simple, cheap, and highly specific and sensitive, but commercial kits for IFAT are supplied without cost by the Brazilian Ministry of Health. Change of the antigens used in serological methods can improve sensitivity and eliminate false-positive results [20]. Perhaps the use of an antigen based on a regional strain of Leishmania may provide better results.

In the present study, IFAT was highly sensitive and was found to be a suitable alternative to parasite detection in the conclusive diagnosis of visceral leishmaniasis in pediatric patients when strong clinical suspicion is present, although in our setting parasite detection is favored by health professionals. In fact, the least requested method for diagnosis was serology.

Molecular techniques such as those based on PCR have been compared with traditional methods, with promising results [21]. The present study showed that the association of microscopy and serology can provide diagnosis in $98.5 \%$ of cases. Considering that the majority of cases of visceral leishmaniasis occur in poor and suburban areas of the countries where it is prevalent [2] and where laboratory resources are probably scarce, conventional methods for diagnosis of the disease are still indispensable, and training professionals in basic techniques should be incremented.

No currently available method for visceral leishmaniasis diagnosis exhibits all the desirable characteristics of high sensitivity and specificity, in addition to ease of use and low cost. We conclude that the use of more than one conventional technique in the diagnosis of visceral leishmaniasis in children is recommended in our region while a single sensitive, specific, less invasive method is not available. Using BMA direct examination and IFAT, confirmation of the disease might be possible in nearly $100 \%$ of cases.

\section{Acknowledgments}

Thanks are given to the staff of the laboratories of Parasitology (Geucira Cristaldo and Zélia Soares da Silva) and Immunology (Dr. Iza K.H. Akamine and Carla Roseli dos Santos) of the UFMS, and to the Central Laboratory of Public Health (LAC), especially to Ruth F. F. da Silva.

\section{References}

1. Gontijo C.M.F., Melo M.N. Visceral leishmaniasis in Brazil: current status, challenges and prospects. Rev Bras epidemiol 2004;7:33849.

2. Desjeux P. Leishmaniasis: current situation and new perspectives. Comp Immunol Microbiol Infect Dis 2004;27:305-18.

3. Osman O.F., Oskam L., Zijlstra E.E., et al. Evaluation of PCR for diagnosis of visceral leishmaniasis. J Clin Microbiol 1997;35:2454-7.

4. Lachaud L., Dereure J., Chabbert E., et al. Optimized PCR using patient blood samples for diagnosis and follow-up of visceral Leishmaniasis, with special reference to AIDS patients. J Clin Microbiol 2000;38:236-40.

5. Salotra P., Sreenivas G., Pogue G.P., et al. Development of a speciesspecific PCR assay for detection of Leishmania donovani in clinical samples from patients with kala-azar and post- kala-azar dermal leishmaniasis. J Clin Microbiol 2001;39:849-54.

6. Cortes S., Rolão N., Ramada J., et al. PCR as a rapid and sensitive tool in the diagnosis of human and canine leishmaniasis using Leishmania donovani s.l.- specific kinetoplastid primers . Trans R Soc Trop Med Hyg 2004;98:12-7.

7. De Doncker S., Hutse V., Abdellati S., et al. A new PCR-ELISA for diagnosis of visceral leishmaniasis in blood of HIV-negative subjects. Trans R Soc Trop Méd Hyg 2005;99:25-31.

8. da Silva E.S., Gontijo C.M., Pacheco R. da S., et al. Diagnosis of human visceral leismaniasis by PCR using blood samples spotted on filter paper. Genet Mol Res 2004;3:251-7.

9. Sundar S. Indian kala-azar: better tools needed for diagnosis and treatment. J Postgrad Med 2003;49:29-30.

10. Campos Jr. D. Características clínico-epidemiológicas do Calazar na criança. Estudo de 75 casos. J Pediatr (Rio J) 1995;71:261-5.

11. Rey L.C., Martins C.V., Ribeiro H.B., et al . American visceral leishmaniasis (kala-azar) in hospitalized children from an endemic area. J Pediatr (Rio J) 2005; 81:73-84.

12. Queiroz M.J., Alves J.G., Correia J.B. Leishmaniose visceral: características clínico-epidemiológicas em crianças de área endêmica. J Pediatr (Rio J) 2004;80:141-6.

13. Pastorino A.C., Jacob C.M., Oselka G.W., Carneiro-Sampaio M. M. Leishmaniose visceral: aspectos clínicos e laboratoriais. J Pediatr (Rio J) 2002;78:120-7. 
14. Pedrosa C.M., da Rocha E.M. Aspectos clínicos e epidemiológicos da leishmaniose visceral em menores de 15 anos procedentes de Alagoas, Brasil. Rev Soc Bras Med Trop 2004;37(4):300-4.

15. Bain B. J. Bone marrow aspiration. J Clin Pathol 2001;54:657-63.

16. da Silva M.R., Stewart J. M., Costa C.H. Sensitivity of bone marrow aspirates in the diagnosis of visceral leishmaniasis. Am J Trop Med Hyg 2005;72:811-4.

17. Murray H.W. Treatment of visceral leishmaniasis (kala-azar): a decade of progress and future approaches. Int J Infect Dis 2000;4:158-77.
18. Singh S. New developments in diagnosis of leishmaniasis. Indian $\mathbf{J}$ Med Res 2006;123(3):311-30.

19. Singh S., Sivakumar R. Recent advances in the diagnosis of leishmaniasis. J Postgrad Med 2003;49(1):55-60.

20. Kar K. Serodiagnosis of leishmaniasis. Crit Rev Microbiol 1995;21:123-52.

21. Gatti S., Gramegna M., Klersy C., et al. Diagnosis of visceral leishmaniasis: the sensitivities and specificities of traditional methods and a nested PCR assay. Ann Trop Med Parasitol 2004;98:667-76. 